This article was published as: ournal of Magnetism and Magnetic Materials 477, 136-141, 2019

DOI: https://doi.org/10.1016/j.jmmm.2019.01.015 


\title{
Shear modulus of isotropic ferrogels
}

\author{
A. Yu.Zubarev ${ }^{a, b}$, A.Yu.Musikhin ${ }^{a}$, M.T. Lopez-Lopez ${ }^{c, d}$, L.Yu. Iskakova $^{\text {a }}$, S.V.Bulytcheva ${ }^{e}$ \\ ${ }^{a}$ Ural Federal University, Lenina Ave 51, 620083, Ekaterinburg, Russia \\ ${ }^{b}$ M.N. Mikheev Institute of Metal Physics of the Ural Branch of the Russian Academy of \\ Sciences, Ekaterinburg, Russia \\ ${ }^{c}$ Departamento de Física Aplicada, Facultad de Ciencias, Universidad de Granada, Campus de \\ Fuentenueva, 18071 Granada, Spain \\ ${ }^{d}$ Instituto de Investigatacion Biosantariaibs. Granada, Spain \\ ${ }^{e}$ Nosov Magnitogorsk State Technical University, Lenin str. 38, 455000, Magnitogorsk, Russia
}

\begin{abstract}
We present results of theoretical study of magnetorheological effect in ferrogels with magnetizable spherical particles chaotically distributed in a current gel. To avoid intuitive constructions with uncontrolled accuracy and adequacy, the analysis is done in the frames of the mathematically regular pair approximation. Our results demonstrate non monotonic increase of the composite shear modulus with the applied magnetic field. This effect is stronger for the systems with the soft gel, than for the relatively rigid ones.
\end{abstract}

\section{Introduction}

Ferrogels are modern smart materials, consisting of magnetic nano - or micron-sized magnetic particles distributed in a polymer matrix. Combination of rich set of properties of polymer materials with a high response to applied magnetic field offers great opportunities in the various high-tech areas, such as magnetically controlled dampers, shock absorbers, sensors, artificial muscles, scaffolds for growing and engineering of biological tissues, etc. [1-14].

One of the most interesting, from the scientific point of view, and valuable from the practical viewpoint, features of these materials is their ability to change shape, size and rheological properties under the action of an external magnetic field. Analysis shows that these magnetomechanic effects strongly depend on the morphology of internal spatial disposition of the particles in the host polymer. The shear effects in the composites with the particles, united in various anisotropic heterogeneous structures, have been studied in refs. [15-18]. The general conclusion of these works is that the field can significantly increase the shear elastic modulus of these composites.

Usually the anisotropic internal structures are created on the stage preceding the composite curing, by application of an external magnetic field (field of polymerization)to the 
suspension of the magnetic particles in the liquid polymer. At the same time, for many applications (especially the bio-medical ones) magnetic gels are synthesized without the field[12]. In this case the spatial disposition of the particles is rather random and isotropic (see, for example, [19-21]).

The goal of this work is theoretical study of influence of an external magnetic field on the shear elastic modulus of magnetic gels with chaotic (without heterogeneous structures)spatial disposition of non Brownian magnetizable particles. The non-linear dependence of the particles magnetization on the field is in focus of our consideration.

\section{Physicalmodel}

The main problem of the theory of composite material is analysis of cooperative effect of interaction between many particles of the filler. To this end various empirical and semi-empirical approaches have been suggested (see, for example, ref.[22]) to estimate mechanical properties of composites with solid non magnetic particles. Unfortunately, these methods do not allow taking into account magnetic interactions between the particles and important details of their spatial disposition in the macroscopically deformed ferrogels.

Here, in order to avoid intuitive and heuristic constructions, we will use mathematically regular approximation of the pair interaction between the particles. In the other words we will take into account magnetic and elastic interactions between two arbitrary particles and ignore any effects of a third one. In the mechanics of suspensions and composite materials this approach, as a rule, leads to quite acceptable agreement with experiments till the particles volume concentrations about $15-20 \%$ [23,24].

Let us consider two identical non Brownian magnetizable particles and denote diameter of the particle as $d_{p}$, radius-vector, linking centers of the particles as $\boldsymbol{r}$. The particles are situated in an elastic incompressible medium with the shear modulus $G_{0}$. The composite is placed in a homogeneous magnetic field $\boldsymbol{H}_{0}$ perpendicular to the direction of the macroscopic shear. We will introduce the Cartesian coordinate system, shown in Fig.1, with the origin in the center of one (say, the first) of the particles, axes $O z$ and $O x$ aligned along the applied field $\boldsymbol{H}_{0}$ and direction of the shear respectively. 


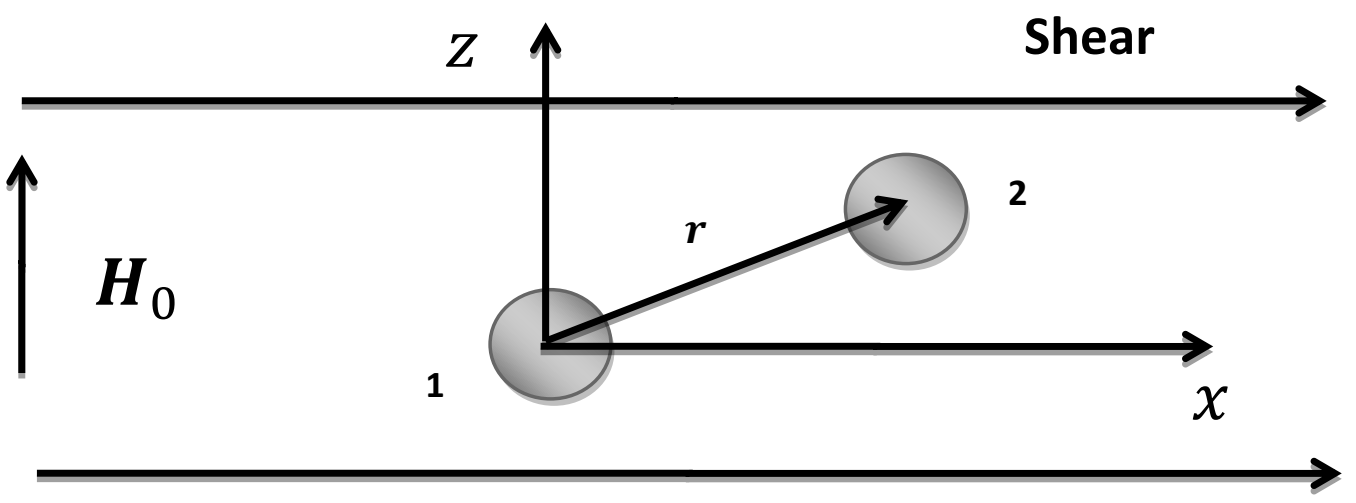

Fig.1.Sketch of the particles relative disposition and used coordinate system. The axis $O y$ is not shown for brevity.

We will take into account magnetic interaction between the particles in the frame of the simplest dipole-dipole interaction. In this approximation the first particle can be considered as placed in the total magnetic field $\boldsymbol{H}=\boldsymbol{H}_{0}+\boldsymbol{H}_{2}$, where

$$
\boldsymbol{H}_{2}=\frac{V_{p}}{4 \pi r^{5}}(3 \cdot(\boldsymbol{M} \cdot \boldsymbol{r}) \boldsymbol{r}-\boldsymbol{M} \cdot \boldsymbol{r})
$$

is the field, created by the second particle in the place where the first one is situated, $\boldsymbol{M}$ is the particle magnetization, $V_{p}=\pi d_{p}^{3} / 6$ isits volume. Since the particles are identical, their magnetizations $\boldsymbol{M}$ are also identical.

Magnetic field $\boldsymbol{H}_{\text {in }}$ inside the particle can be found from the following relation [25]:

$$
\boldsymbol{H}_{0}+\boldsymbol{H}_{2}=\boldsymbol{H}_{\text {in }}+N \cdot \boldsymbol{M}
$$

where $N=1 / 3$ - is demagnetizing factor of the spherical particle. In its turn, magnetization $\boldsymbol{M}$ of the particle can be estimated by using the empirical Frolich-Kennelly relation $[26,27]$ :

$$
\boldsymbol{M}=\chi \cdot \boldsymbol{H}_{\text {in }}, \quad \chi=\frac{\chi_{0} M_{\text {sat }}}{M_{\text {sat }}+\chi_{0}\left|\boldsymbol{H}_{\text {in }}\right|} .
$$

Here $\chi_{0}$ and $M_{\text {sat }}$ are initial susceptibility of the particle material and its saturated magnetization respectively; $\chi$ is the particle susceptibility in the internal field $\boldsymbol{H}_{i n}$. Combining eqs. (2) and (3), one gets: 


$$
\boldsymbol{H}_{0}+\boldsymbol{H}_{2}=\boldsymbol{H}_{\text {in }} \cdot\left(1+\frac{1}{3} \cdot \frac{\chi_{0} M_{\text {sat }}}{M_{\text {sat }}+\chi_{0}\left|\boldsymbol{H}_{\text {in }}\right|}\right) .
$$

Substituting here the relation (1), taking into account (3), we come to the system of equations with respect to the components of magnetization $\boldsymbol{M}$ :

$$
\begin{aligned}
& M_{x}=\left[1-\frac{6 M_{\text {sat }}}{3 \chi_{0} H^{\prime}+M_{\text {sat }}\left(3-\chi_{0}\right)+\sqrt{\left(M_{\text {sat }}\left(3-\chi_{0}\right)+3 \chi_{0} H^{\prime}\right)^{2}+12 \chi_{0} M_{\text {sat }}^{2}}}\right] \frac{M_{s a t} H_{2 x}}{H^{\prime}}, \\
& M_{y}=\left[1-\frac{6 M_{\text {sat }}}{3 \chi_{0} H^{\prime}+M_{\text {sat }}\left(3-\chi_{0}\right)+\sqrt{\left(M_{\text {sat }}\left(3-\chi_{0}\right)+3 \chi_{0} H^{\prime}\right)^{2}+12 \chi_{0} M_{\text {sat }}^{2}}}\right] \frac{M_{\text {sat }} H_{2 x}}{H^{\prime}}, \\
& M_{z}=\left[1-\frac{6 M_{\text {sat }}}{3 \chi_{0} H^{\prime}+M_{\text {sat }}\left(3-\chi_{0}\right)+\sqrt{\left(M_{\text {sat }}\left(3-\chi_{0}\right)+3 \chi_{0} H^{\prime}\right)^{2}+12 \chi_{0} M_{\text {sat }}^{2}}}\right] \frac{M_{\text {sat }}\left(H_{2 z}+H_{0 z}\right)}{H^{\prime}} \text {, } \\
& \text { where } H^{\prime}=\sqrt{H_{2 x}{ }^{2}+H_{2 y}{ }^{2}+\left(H_{0 z}+H_{2 z}\right)^{2}} \text {. }
\end{aligned}
$$

It will be convenient to use the spherical coordinate system with the radius $r$,polar and azimuthal angles $\theta$ and $\phi$, defined so that $\left(H_{0 z}\right.$ will be denoted as $\left.H_{0}\right)$ :

$$
x=r \sin \theta \cos \phi, y=r \sin \theta \sin \phi, z=r \cos \theta,
$$

in this coordinates system one gets

$$
\begin{aligned}
& M_{x}\left(\rho, \theta, \phi, H_{0}\right)=\frac{8}{3} M\left(\rho, H_{0}\right) \sin \theta \cos \theta \cos \phi, \\
& M_{y}\left(\rho, \theta, \phi, H_{0}\right)=\frac{8}{3} M\left(\rho, H_{0}\right) \sin \theta \cos \theta \sin \phi, \\
& M_{z}\left(\rho, \theta, \phi, H_{0}\right)=M_{z}\left(\rho, \theta, H_{0}\right) .
\end{aligned}
$$

Here $\rho=2 r / d_{p}$ is dimensionless distance between the particles. Some results of calculations of the components $M_{x}, M_{y}, M_{z}$ are shown in Fig. $2 ; M\left(\rho, H_{0}\right)$ is absolute value of the particle magnetization, is to be determined. 


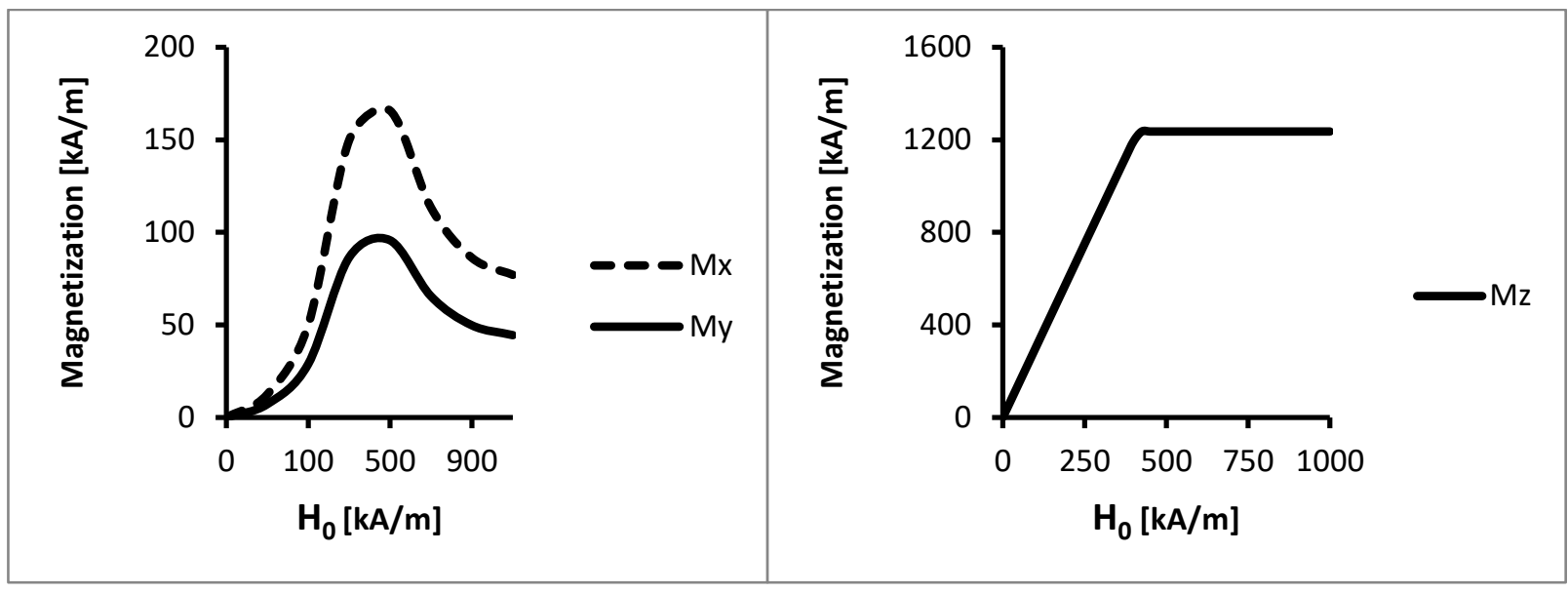

Fig.2.The components $M_{x}, M_{y}, M_{z}$ vs. the applied field $H_{0}$ for $\rho=2, \theta=\frac{\pi}{3}, \phi=\frac{\pi}{6}$ and $d_{p}=$

$$
1 \mu m, \chi_{0}=15000, M_{\text {sat }}=1245 \frac{\mathrm{kA}}{\mathrm{m}} \text {. }
$$

Note that the components $M_{x}, M_{y}$ non monotonic, with maximums, depend on the field $\boldsymbol{H}_{0}$, parallel to the axis $O z$.

Now we are in position to determine effect of the field $\boldsymbol{H}_{\mathbf{0}}$ on the shear modulus $G$ of the composite. We suppose that the system experiences macroscopic shear along the axis $O x$ with the displacement $u_{x}=\gamma z$, where $z$ is the Cartesian coordinate, $\gamma$ is the dimensionless shear.

The component $\sigma_{x z}$ of the macroscopic (measurable) stress tensor $\boldsymbol{\sigma}$ in the composite can be presented as $[22,25,28]$ :

$$
\begin{aligned}
& \sigma=\sigma_{x z}=\sigma_{m}+\sigma_{e l}=G_{m} \gamma+G_{e l} \gamma, \quad \text { or } G=G_{e l}+G_{m} \\
& G_{m} \gamma=\frac{1}{2} \varphi \mu_{0}<M_{x}>H_{0} .
\end{aligned}
$$

Here $\sigma_{m}$ is the part of the total stress, induced by the applied field, $\varphi$ is volume concentration of the particles; $\mu_{0}$ is the vacuum magnetic permeability; $\left\langle M_{x}\right\rangle$ ismean component of the particle magnetization averaged over all positions of the second particle; $\sigma_{e l}$ is the elastic shear stress of the composite with the hard non magnetic spheres; $G_{e l}$ is the corresponding elastic modulus of the composite. We will estimate it by using the well known Batchelor-Green formula [23]

$$
G_{e l}=G_{0}\left(1+2.5 \varphi+5.2 \varphi^{2}\right)
$$

where $G_{0}$ isthe shear modulus of the pure host polymer. Usually this formula leads to quite acceptable agreement with experiments in the range of concentration till $\varphi \sim 15-25 \%$ [23]. 
In order to determine the total elastic modulus, we must determine the magnitude $\left\langle M_{x}\right\rangle$. To this end let us introduce the pair function $g(\boldsymbol{r})$ of the particles spatial disposition in thehost matrix, normalized as $\lim _{r \rightarrow \infty} g(\boldsymbol{r})=1$. In the approximation of the pair interaction between the particles, the $x$-component of the mean magnetization can be presented as

$$
<M_{x}>=\frac{\varphi}{V_{p}} \int M_{x}(\boldsymbol{r}) g(\boldsymbol{r}) d \boldsymbol{r},
$$

The distribution function can be written down in the form:

$$
g(\boldsymbol{r})=g_{0}(\boldsymbol{r})+\delta g(\boldsymbol{r})
$$

where $g_{0}(\boldsymbol{r})$ is the function in the non deformed composite before the field application; $\delta g(\boldsymbol{r})$ is change of the distribution function, corresponding to the rearrangement of the particles because of their magnetic interaction and macroscopic deformation of the sample.
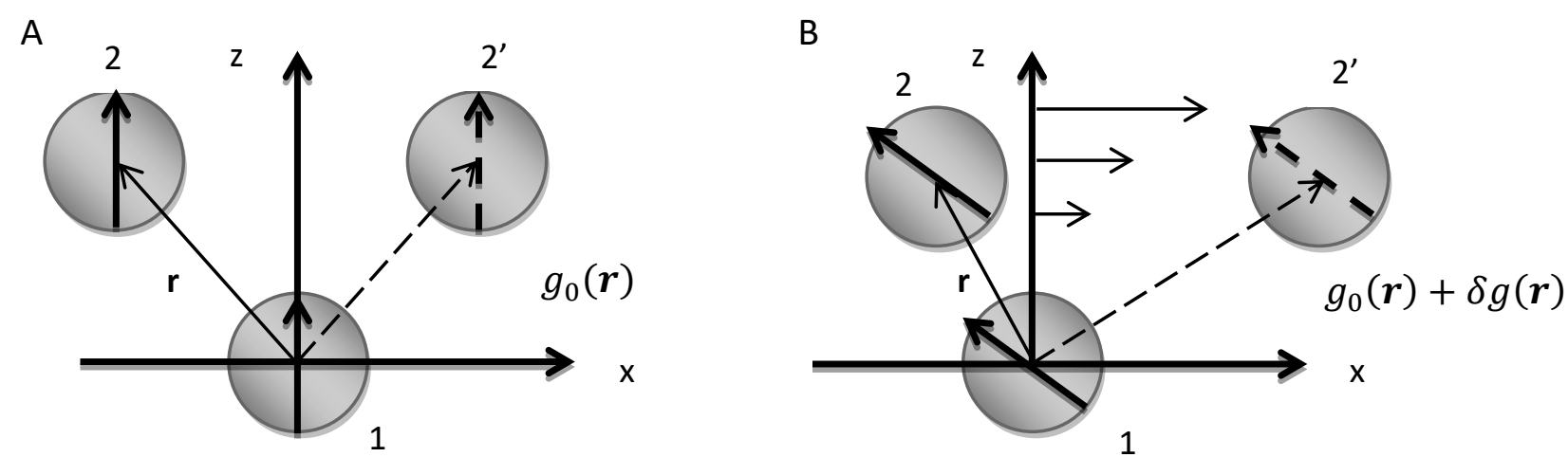

Fig.3. Sketch of change of the relative distribution of the particles as a consequence of the macroscopic shear of the composite. A - isotropic distribution of the particles in the non deformed composite;

$$
\mathrm{B} \text { - after the shear deformation. }
$$

We will use $g_{0}(\boldsymbol{r})$ in the simplest form

$$
g_{0}(\rho)=\left\{\begin{array}{c}
0, \rho<2 \\
1+8 \varphi\left(1-\frac{3 \rho}{2^{3}}+\frac{3 \rho^{3}}{2^{7}}\right), \quad 2<\rho<4 \\
1, \rho>4
\end{array}\right.
$$

here $\rho=2 r / d_{p} \quad$ is dimensionless distance between the particles. This form includes effect of the short-ranged order of hard spheres spatial disposition [29]. 
Substitutingeq.(10) into (9), taking into account that in an isotropic composite the equality $\int M_{x}(\boldsymbol{r}) g_{0}(\boldsymbol{r}) d \boldsymbol{r}=0$ is held, we get

$$
<M_{x}>=\frac{\varphi}{V} \int M_{x}(\boldsymbol{r}) \delta g(\boldsymbol{r}) d \boldsymbol{r}
$$

The function $\delta g(\boldsymbol{r})$ can be determined from the equation [30]

$$
\delta g(\boldsymbol{r})=-\operatorname{div}(g(\boldsymbol{r}) \boldsymbol{w})
$$

where $\boldsymbol{w}$ is vector of relative displacement of the particles.

\section{Particles rearrangement because of magnetic interaction}

Let us consider the non sheared composite placed into the field $\boldsymbol{H}_{0}$. In the frame of the dipole-dipole approximation, the energy of magnetic interaction between the particles has the form:

$$
U(r)=-\frac{\mu_{0} V_{p}^{2}}{4 \pi}\left[3 \frac{(\boldsymbol{M} \cdot \boldsymbol{r})^{2}}{r^{5}}-\frac{\boldsymbol{M}^{2}}{r^{3}}\right]
$$

The force o fmagnetic interaction between the particles is $\boldsymbol{F}=-\nabla U(r)$. After simple calculations, one can get

$$
\begin{aligned}
& F_{x}=\frac{3 \mu_{0} V_{p}^{2}}{4 \pi r^{5}}\left(2 M_{x}\left(M_{x} x+M_{y} y+M_{z} z\right)-\frac{5 x\left(M_{x} x+M_{y} y+M_{z} z\right)^{2}}{r^{2}}+x\left(M_{x}{ }^{2}+M_{y}{ }^{2}+M_{z}{ }^{2}\right)\right), \\
& F_{y}=\frac{3 \mu_{0} V_{p}^{2}}{4 \pi r^{5}}\left(2 M_{y}\left(M_{x} x+M_{y} y+M_{z} z\right)-\frac{5 y\left(M_{x} x+M_{y} y+M_{z} z\right)^{2}}{r^{2}}+y\left(M_{x}{ }^{2}+M_{y}{ }^{2}+M_{z}{ }^{2}\right)\right), \\
& F_{z}=\frac{3 \mu_{0} V_{p}^{2}}{4 \pi r^{5}}\left(2 M_{z}\left(M_{x} x+M_{y} y+M_{z} z\right)-\frac{5 z\left(M_{x} x+M_{y} y+M_{z} z\right)^{2}}{r^{2}}+z\left(M_{x}{ }^{2}+M_{y}{ }^{2}+M_{z}{ }^{2}\right)\right) .
\end{aligned}
$$

Vector of the relative displacement $\boldsymbol{w}_{\boldsymbol{m}}$, induced by the magnetic interaction between the particles, can be determined as:

$$
w_{m}=\widehat{\boldsymbol{\beta}} \cdot \boldsymbol{F},
$$


Here $\widehat{\boldsymbol{\beta}}$ is the tensor of the matrix elastic resistance to the particles displacement, which has the following form [24] :

$$
\begin{aligned}
& \beta_{i i}=\beta_{0}\left(\left(G_{B}(\rho)-H_{B}(\rho)\right) \frac{x_{i}{ }^{2}}{r^{2}}+H_{B}(\rho)\right), \\
& \beta_{i j}=\beta_{0}\left(G_{B}(\rho)-H_{B}(\rho)\right) \frac{x_{i} x_{j}}{r^{2}} \\
& \beta_{0}=\frac{2}{3 \pi G_{0} d_{p}} .
\end{aligned}
$$

Here $i, j=x, y, z ; G_{B}(\rho)$ and $H_{B}(\rho)$ presen tsome functions of the dimensionless distance $\rho$ between the particles centers; their numerical values are tabulated in [24]. Since explicit analytical forms of these functions are unknown, we will use the following extrapolation formulas, suggested in [31]

$$
\begin{aligned}
& G_{B}(\rho)=\frac{2(\rho-2)\left|1-\frac{3}{2} \rho^{-1}+\rho^{-3}-\frac{15}{4} \rho^{-4}\right|}{2(\rho-2)+\left|1-\frac{3}{2} \rho^{-1}+\rho^{-3}-\frac{15}{4} \rho^{-4}\right|}, \\
& H_{B}(\rho)=\frac{0.401(\rho-1)}{0.401(\rho-2)+1} .
\end{aligned}
$$

Combining eqs.(15-17), one can find the displacement vector $\boldsymbol{w}_{m}$. In the spherical coordinate system (6), the components of this vector read:

$$
\begin{aligned}
& w_{m r}=-\pi d_{p}^{2} \beta_{0} \mu_{0}\left(\frac{G_{B}(\rho)}{\rho^{4}}\left[M^{2}(\rho) X X_{r}(\theta)+M_{z}\left(\rho, \theta, H_{0}\right) M(\rho) X Z_{r}(\theta)+M_{z}^{2}\left(\rho, \theta, H_{0}\right) Z Z_{r}(\theta)\right]\right), \\
& w_{m \theta}=-\pi d_{p}^{2} \beta_{0} \mu_{0}\left(\frac{H_{B}(\rho)}{\rho^{4}}\left[M^{2}(\rho) X X_{\theta}(\theta)+M_{z}\left(\rho, \theta, H_{0}\right) M(\rho) X Z_{\theta}(\theta)+M_{z}^{2}\left(\rho, \theta, H_{0}\right) Z Z_{\theta}(\theta)\right]\right), \\
& w_{m \phi}=0 .
\end{aligned}
$$

Here

$$
\begin{aligned}
& X X_{r}(\theta)=\frac{4}{27}\left(2 \sin ^{2} 2 \theta-3 \sin ^{2} 3 \theta+3 \sin ^{2} \theta\right) ; X X_{\theta}(\theta)=-\frac{16}{27} \sin ^{3} 2 \theta \\
& X Z_{r}(\theta)=\frac{4}{3} \sin ^{2} 2 \theta ; \quad X Z_{\theta}(\theta)=-\frac{4}{9} \sin 4 \theta ; \\
& Z Z_{r}(\theta)=\frac{2-3 \sin ^{2} \theta}{3} ; \quad Z Z_{\theta}(\theta)=\frac{1}{3} \sin 2 \theta
\end{aligned}
$$

By using the spherical coordinate system (6), one can rewrite eq.(13) as: 


$$
\delta g_{m}(\boldsymbol{r})=-\operatorname{div}\left(g_{0}(\boldsymbol{r}) \boldsymbol{w}_{m}\right)=-\frac{2}{d_{p}}\left[\frac{1}{\rho^{2}} \frac{\partial}{\partial \rho}\left(w_{m r} g_{0}(\rho) \rho^{2}\right)+\frac{g_{0}(\rho)}{\rho \sin \theta} \frac{\partial}{\partial \theta}\left(w_{m \theta} \sin \theta\right)\right]
$$

Combining eqs.(19,20), we find the explicit form of $g_{m}(\boldsymbol{r})$. This function reflects the anisotropy, which appears in the relative disposition of the particles because of their magnetic interaction and elastic resistance of the carrier medium to the particles displacement.

\section{The structural anisotropy due to the shear deformation}

Now we will take into account the macroscopic shear deformation of the composite, illustrated in Fig.1. Let us introduce the vector $\boldsymbol{w}_{\gamma}$ of the particles relative displacement, induced by this deformation. According to [22, 23], the spherical components of this displacement can be written down as

$$
\begin{gathered}
w_{\gamma r}=\frac{d_{p}}{2} \gamma\left(\rho\left(1-A_{B}(\rho)\right) \sin \theta \cos \theta \cos \phi\right) \\
w_{\gamma \theta}=\frac{d_{p}}{2} \gamma\left(\rho\left(\cos ^{2} \theta+\frac{1}{2} B_{B}(\rho)\left(\sin ^{2} \theta-\cos ^{2} \theta\right)\right) \cos \phi\right) \\
w_{\gamma \phi}=\frac{d_{p}}{2} \gamma\left(\rho\left(1-\frac{1}{2} B_{B}(\rho)\right) \cos \theta \sin \phi\right) .
\end{gathered}
$$

Here $\gamma$ is the dimensionless shear, $A_{B}(\rho) \operatorname{and} B_{B}(\rho)$ are functions, whose numerical values are tabulated [23]. Explicit analytical forms of these functions are unknown. The following extrapolations have been suggested in [31]:

$$
\begin{aligned}
& A_{B}(\rho)=\left\{\begin{array}{l}
1-4.077(\rho-2), \quad \text { if } 2<\rho<2.13 \\
5 \rho^{-3}-\frac{40}{3} \rho^{-5}+25 \rho^{-6}, \text { if } \rho>2.13^{\prime}
\end{array}\right. \\
& B_{B}(\rho)=\frac{0.406\left(\frac{16}{3}\right)(2 \rho)^{-5}}{\left(\frac{16}{3}\right)(2 \rho)^{-5}+0.406\left(2^{-5}-\rho^{-5}\right)}
\end{aligned}
$$

The change $\delta g_{\gamma}(\boldsymbol{r})$ of the distribution function, provoked by the macroscopic deformation of the composite, can be found from eq. (13):

$$
\begin{aligned}
& \delta g_{\gamma}(\boldsymbol{r})=-\operatorname{div}\left(\left(g_{0}(\boldsymbol{r})+\delta g_{m}(\boldsymbol{r})\right) \boldsymbol{w}_{\gamma}\right)=\delta g_{\gamma}(\boldsymbol{r})^{(1)}+\delta g_{\gamma}(\boldsymbol{r})^{(2)}, \\
& \delta g_{\gamma}(\boldsymbol{r})^{(1)}=-\operatorname{div}\left(g_{0}(\boldsymbol{r}) \boldsymbol{w}_{\gamma}\right),
\end{aligned}
$$




$$
\delta g_{\gamma}(\boldsymbol{r})^{(2)}=-\operatorname{div}\left(\delta g_{m}(\boldsymbol{r}) \boldsymbol{w}_{\gamma}\right)
$$

The term $\delta g_{\gamma}(\boldsymbol{r})^{(1)}$ reflects the relative rearrangement of the particles, induced only by the shear deformation of the composite. The term $\delta g_{\gamma}(\boldsymbol{r})^{(2)}$ corresponds to cooperative effect of the magnetic interaction between the particles and the composite macroscopic shear deformation.

\section{Averaged components of the particle magnetization}

The total change $\delta g(\boldsymbol{r})$ of the pair distribution function can be presented as

$$
\delta g(\boldsymbol{r})=\delta g_{m}(\boldsymbol{r})+\delta g_{\gamma}(\boldsymbol{r})
$$

Substituting (24) into (12) and taking into account tha t $\int M_{x}(\boldsymbol{r}) \delta g_{m}(\boldsymbol{r}) d \boldsymbol{r}=0$, one can get:

$$
\begin{aligned}
& <M_{x}>=<M_{x}>^{(1)}+<M_{x}>^{(2)}, \\
& <M_{x}>^{(1)}=\frac{\varphi}{V} \int M_{x}(\boldsymbol{r}) \delta g_{\gamma}^{(1)}(\boldsymbol{r}) d \boldsymbol{r}, \\
& <M_{x}>^{(2)}=\frac{\varphi}{V} \int M_{x}(\boldsymbol{r}) \delta g_{\gamma}^{(2)}(\boldsymbol{r}) d \boldsymbol{r} .
\end{aligned}
$$

By using here the relations (5),(6), (21) and (23), after simple, but cumbersome calculations, we come to the following relations:

$$
\begin{aligned}
& <M_{x}>^{(1)}=-\frac{8 \varphi \gamma}{15} J\left(\varphi, H_{0}\right) \\
& J\left(\varphi, H_{0}\right)=\int_{0}^{\infty} M\left(\rho, H_{0}\right)\left[\frac{d}{d \rho}\left(\rho^{3}\left(1-A_{B}(\rho)\right) g_{0}(\rho)\right)-3 \rho^{2} g_{0}(\rho)\left(1-B_{B}(\rho)\right)\right] d \rho .
\end{aligned}
$$

In order to calculate $<M_{x}>^{(2)}$ let us present $\delta g_{\gamma}(\boldsymbol{r})^{(2)}$ in the form

$$
\begin{aligned}
\delta g_{\gamma}(\boldsymbol{r})^{(2)}=- & \frac{2}{d_{p}}\left[\frac{1}{\rho^{2}} \frac{\partial}{\partial \rho}\left(\delta g_{m}(\boldsymbol{r}) w_{\gamma r} g_{0}(\rho) \rho^{2}\right)+\frac{g_{0}(\rho)}{\rho \sin \theta} \frac{\partial}{\partial \theta}\left(\delta g_{m}(\boldsymbol{r}) w_{\gamma \theta} \sin \theta\right)\right. \\
& \left.+\frac{g_{0}(\rho)}{\rho \sin \theta} \frac{\partial}{\partial \phi}\left(\delta g_{m}(\boldsymbol{r}) w_{\gamma \phi}\right)\right]
\end{aligned}
$$

which directly follows from eqs. (20) and (23). Here again $w_{\gamma r}, w_{\gamma \theta}, w_{\gamma \phi}$ are spherical coordinates of the vector $\boldsymbol{w}_{\gamma}$. 
Combining (25) and (27), one gets after transformations:

$$
\begin{aligned}
<M_{x}>^{(2)}= & 4 d_{p} \gamma \varphi \beta_{0} \mu_{0} K\left(\varphi, H_{0}\right), \\
K\left(\varphi, H_{0}\right)= & \frac{-1}{\gamma \pi d_{p}^{2} \beta_{0} \mu_{0}} \int_{0}^{2 \pi} \int_{0}^{\pi} \int_{0}^{\infty} M\left(\rho, H_{0}\right) \sin ^{2} \theta \cos \theta \cos \phi\left[\frac{\partial}{\partial \rho}\left(\delta g_{m}(\boldsymbol{r}) w_{\gamma r} g_{0}(\rho) \rho^{2}\right)\right. \\
& \left.+\frac{g_{0}(\rho) \rho}{\sin \theta} \frac{\partial}{\partial \theta}\left(\delta g_{m}(\boldsymbol{r}) w_{\gamma \theta} \sin \theta\right)+\frac{g_{0}(\rho) \rho}{\sin \theta} \frac{\partial}{\partial \phi}\left(\delta g_{m}(\boldsymbol{r}) w_{\gamma \phi}\right)\right] d \rho d \theta d \phi .
\end{aligned}
$$

The integrals $J\left(\varphi, H_{0}\right)$ and $K\left(\varphi, H_{0}\right)$ can be calculated numerically.

\section{Results and discussions}

Having the mean magnetization component $\left\langle M_{x}\right\rangle$ determined, one can find the macroscopic shear modulus $G$ of the composite. Taking into account (7) and (25), we get

$$
\begin{aligned}
& \sigma=\sigma_{e l}+\sigma_{m}{ }^{(1)}+\sigma_{m}{ }^{(2)}, \\
& \sigma_{m}{ }^{(1)}=G_{m}{ }^{(1)} \gamma=\frac{1}{2} \varphi \mu_{0}<M_{x}>^{(1)} H_{0}, \\
& \sigma_{m}{ }^{(2)}=G_{m}{ }^{(2)} \gamma=\frac{1}{2} \varphi \mu_{0}<M_{x}>^{(2)} H_{0} .
\end{aligned}
$$

Here $\sigma_{m}^{(1)}$ and $\sigma_{m}^{(2)}$ are the magnetically induced parts of the total stress $\sigma$, which appear due to the change of the particles mutual disposition, as a consequence of the macroscopic shear deformation of the isotropic composite and because of combination of this deformation with the magnetically induced particles rearrangement, respectively. Combining (7) and (28), one comes to the relation

$$
\begin{gathered}
G=G_{e l}+G_{m}, \\
G_{m}=G_{m}{ }^{(1)}+G_{m}{ }^{(2)} .
\end{gathered}
$$

Here $G_{m}$ is magnetically induced part of the total shear modulus of the composite, parameters $G_{m}^{(1)}$ and $G_{m}^{(2)}$ are the parts of the magnetic contributions to the total shear modulus $G$, corresponding to $\sigma_{m}^{(1)}$ and $\sigma_{m}^{(2)}$ respectively.

Taking into account $(26,28,29)$ a s well as relation (17) for $\beta_{0}$, one gets the relations

$$
\begin{aligned}
G_{m}{ }^{(1)} & =-\frac{4}{15} \varphi^{2} \mu_{0} J\left(\varphi, H_{0}\right) H_{0}, \\
G_{m}{ }^{(2)} & =\frac{4}{3 \pi} \frac{\varphi^{2} \mu_{0}{ }^{2}}{G_{0}} K\left(\varphi, H_{0}\right) H_{0} .
\end{aligned}
$$


Let us introduce the dimensionless parameters:

$$
h=\frac{H_{0}}{M_{\text {sat }}}, \quad j=\frac{J\left(\varphi, H_{0}\right)}{H_{0}}, k=\frac{K\left(\varphi, H_{0}\right)}{H_{0}{ }^{3}} \text { and } G^{\prime}=\frac{G}{G_{0}} .
$$

Note that $J \sim H_{0}, K \sim H_{0}{ }^{3}$ when $H_{0} \rightarrow 0$. Therefore the parameters $j$ and $k$ tend to some finite values when the field $H_{0}$ tends to zero.

By using these notifications, combining eqs.(8), (30) and (31), we come to the final formula for the shear modulus:

$$
\begin{gathered}
G=Q G_{0}, Q=Q_{e l}+Q_{m} \text { and } Q_{e l}=1+2.5 \varphi+5.2 \varphi^{2} . \\
Q_{m}=-\frac{4 \varphi^{2}}{15} \frac{\mu_{0} M_{s a t}^{2} h^{2}}{G_{0}} j+\frac{4 \varphi^{2}}{3 \pi}\left(\frac{\mu_{0} M_{s a t}^{2} h^{2}}{G_{0}}\right)^{2} k .
\end{gathered}
$$

Here $Q$ is dimensionless shear modulus of the composite, $Q_{e l}$ and $Q_{m}$ are its elastically and magnetically induced parts.

Some results of calculations of the magnetic part $Q_{m}$ are presented in Figs.4-6. For all magnitudes of the dimensionless field $h$, presenting interest, $Q_{m}$ is positive, i.e. the second term in the relation for $Q_{m}$ dominates over the first one.

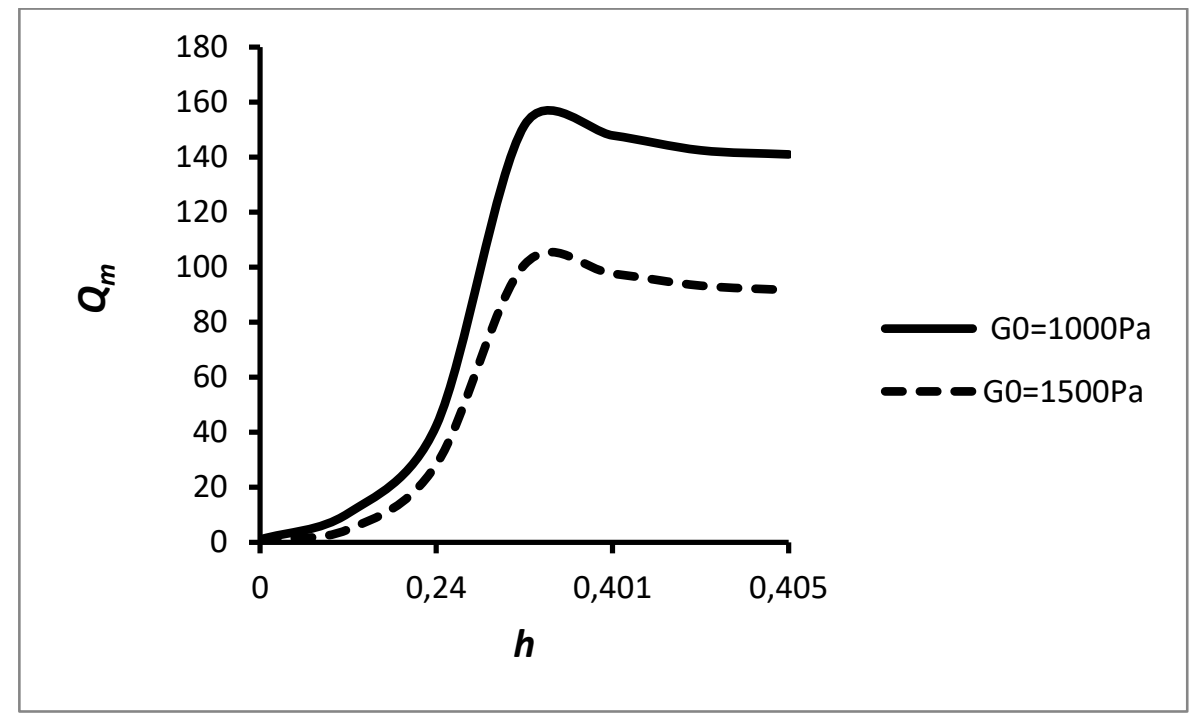

Fig. 4. Dimensionless magnetically induces modulus $Q_{m}$ of the composite vs. dimensionless magnetic field $h$. Parameters of the system: $\varphi=0.1, \chi_{0}=15000, M_{\text {sat }}=1245 \frac{\mathrm{kA}}{\mathrm{m}}$. 


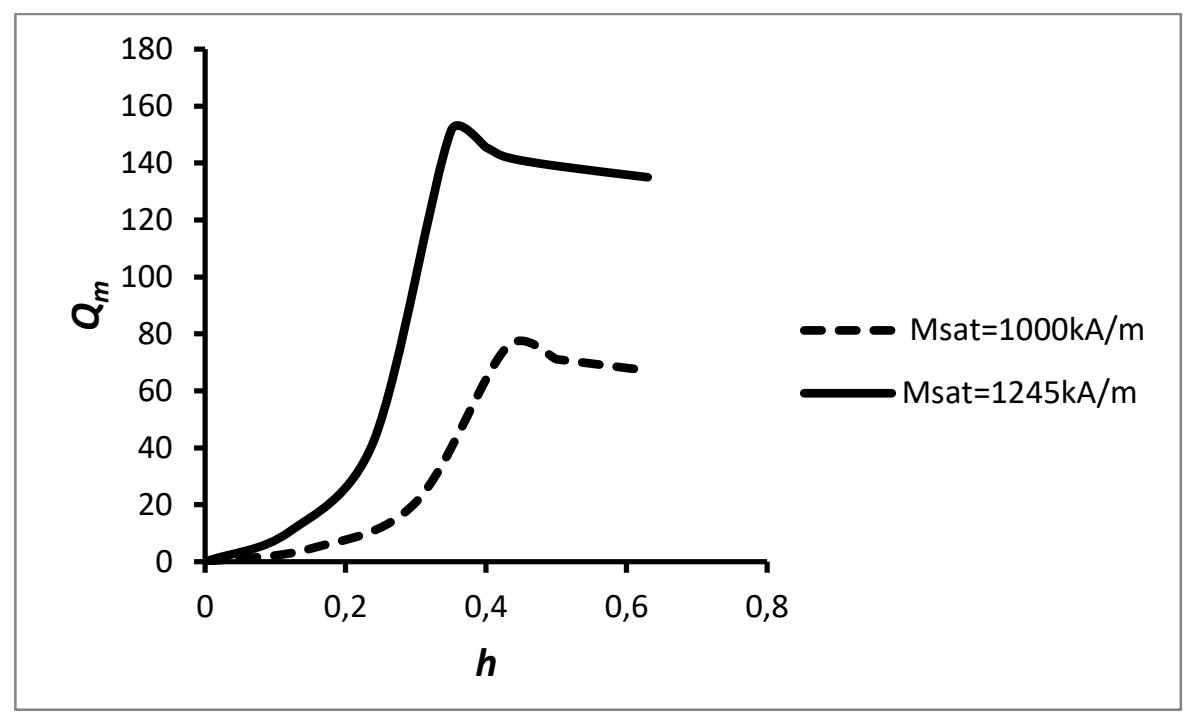

Fig. 5.Same as in Fig. 4 for: $\varphi=0.1, \chi_{0}=15000, G_{0}=10^{3} \mathrm{~Pa}$.

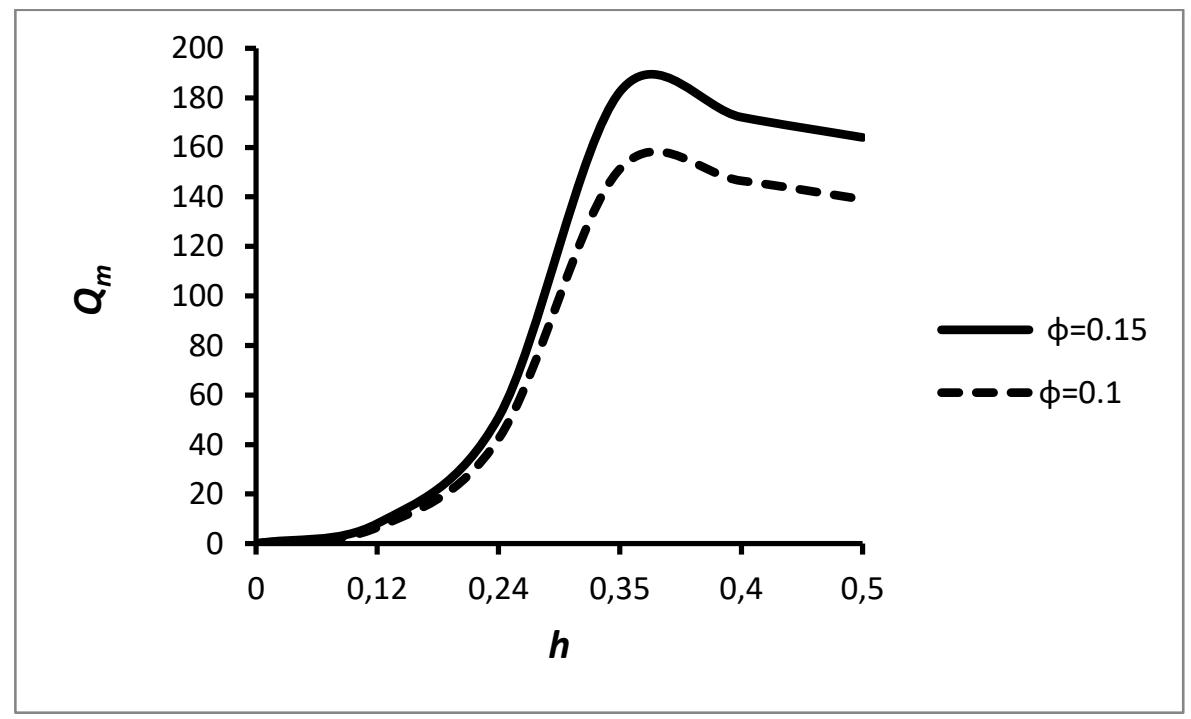

Fig. 6. Same as in Figs. 4,5 when $\chi_{0}=15000, M_{\text {sat }}=1245 \frac{\mathrm{kA}}{\mathrm{m}}, G_{0}=10^{3} \mathrm{~Pa}$.

The results show that the modulus $G_{m}$ non monotonic, with maximum, depends on the applied field; when the field tends to infinity, the modulus $G_{m}$ asymptotically tends to some finite magnitude. The non monotonic character of the dependence of $G_{m}$ on $H_{0}$ is explained by the non monotonic dependence of the component $M_{x}$ on the field, illustrated in Fig. 2.

\section{Conclusion}

We present results of theoretical modeling of effect of applied magnetic field on elastic shear modulus of magnetic gel with homogeneous and chaotic spatial distribution of magnetizable non Brownian spherical particles in an elastic medium. Analysis shows that the 
magnetorheological effect appears because of combination of mutual magnetization of the particles and the change the function of their mutual spatial disposition as a consequence of the macroscopic deformation of the composite. In order to avoid intuitive and heuristic theoretical construction with uncontrolledadequacy, the elastic and magnetic interparticle interactions are taken into account in the frames of mathematically regular approximation of the pair interaction.

Our results demonstrate that for strengths of the field, in the range of the ones, used in experiments, magnetic field enhances macroscopic modulus of the composite. The modulus non monotonic, with maximum, depends on the field strength and tends to a certain finite magnitude when the field tends to infinity.

It should be noted that in real experiments, because of features of the composites synthesis, the particles can form various agglomerates, which can mask the effects, predicted by the ideal model. Effect of these agglomerates on the magnetomechanic phenomena in the composites, requires separate study for each concrete situation.

\section{Acknowledgements}

AZ, L.I. and AM is grateful to the program of the Ministry of Education and Science of the Russian Federation, projects 02.A03.21.0006; 3.1438.2017/4.6; 3.5214.2017/6.7 as well as to the Russian Fund of Basic Researches, projects 18-08-00178 and 19-52-12028. M.T.L-L acknowledges financial support by project FIS2017-85954-R (Ministerio de Economía, Industria y Competitividad, MINECO, and Agencia Estatal de Investigación, AEI, Spain, cofunded by Fondo Europeo de Desarrollo Regional, FEDER, European Union).

\section{References}

[1] G. Filipcsei, I. Csetneki, A. Szilagyi, M. Zrınyi, Adv. Polym. Science 206(2007) 137.

[2] A. Boczkowska, S.F. Awietjan, Materials Science Forum 636-637 (2010) 766.

[3] M. van Bruggen, J. van Zon, Sensors and Actuators, A Physics158(2010) 240.

[4] S.van Berkum, J.T. Dee, Albert P. Philipse, B.H. Erné, Int. J. Mol. Science14(2013)10162.

[5] M.Bañobre-López, Y.Piñeiro-Redondo, R. de Santis, A.Gloria, L.Ambrosio, A.Tampieri, et al. J Appl Phys. 109 (2011) 07B313.

[6] B.Das, M.Mandal, A.Upadhyay, P.Chattopadhyay, N. Karak., Biomed. Mater. 8 (2013) 035003.

[7] A.Gloria, R.Russo, U. d'Amora, S. Zeppetelli, T.d'Alessandro, M. Sandri, et al., J. R. SocInterface 10 (2013) 20120833.

[8] Y. Li, G.Huang, X.Zhang, B.Li, Y.Chen, T.Lu, et al, AdvFunct Mater. 23(2013) 660. 
[9] S.Panseri, C.Cunha, T.'Alessandro, M.Sandri, G.Giavaresi, M.Marcacci, et al., J Nanobiotechnology10(2012) 32.

[10] X.B. Zeng, H.Hu, L.Q.Xie, F.Lan, W.Jiang, Y.Wu, et al., Int J Nanomed. 7 (2012) 3365.

[11] R.K.Singh, K.D.Patel, J.H. Lee, E.J.Lee, J.H.Kim, T.H.Kim, et al., PLoSOne9(2014) e91584.

[12] M. T. Lopez-Lopez, G.Scionti, A.C. Oliveira, J.D. G. Duran, A. Campos, M. Alaminos, I.A.Rodriges, PLoS One 10 (2015) e0133878.

[13] M.T. Lopez-Lopez, Juan D.G. Durán, L. Yu. Iskakova,A.Yu. Zubarev, Journal of Nanofluids5 (2016) 1.

[14] R. Weeber, M. Hermes, A.M. Schmidt, C. Holm, J. Phys.: Condens. Matter 30 (2018) 063002.

[15] M.R. Jolly, J.D. Carlson, B.C. Muñoz, T.A. Bullions, Journal of Intelligent Material Systems and Structures 6(1996) 613.

[16] K. Danas, S.V.Kankanala, N.Triantafyllidis, J. Mechanics and Physics of Solids60 (2012) 120.

[17] Y.Han, W.Hong, L.E. Faidley, International Journal of Solids and Structures 50(2013) 2281 .

[18] I. Agirre-Olabide, P. Kuzhir, M.J. Elejabarrieta, J. Magnetism and Magnetic Materials 446(2018) 155-161.

[19] G.V. Stepanov, S.S. Abramchuk, D.A. Grishin, L. V. Nikitin, E.Y. Kramarenko, A.R. Khokhlov, Polymer48(2007) 488.

[20] G.Stepanov, D.Borin, Yu.Raikher, P.Melenev, N.S. Perov, J. Phys.: Condens. Matter 20(2008) 204121.

[21] S.R. Khimi, K.L.Pickering, Compos. Part B Eng.83(2015) 175.

[22] R.Cristensen, Mechanics of Composite Materials, Wiley, New York, 1979.

[23] G.K.Batchelor, J.T.Green, J.Fluid Mechanics56(3) (1972) 4101.

[24] G.K.Batchelor, J.Fluid Mechanics83(1) (1977) 97.

[25] L.D. Landau, E.M. Lifshits, Electrodynamics of Continuous Media, Pergamon Press, 1984.

[26] R.M. Bozorth, Ferromagnetism, Wiley-IEEE Press, 1993.

[27] A.M. Biller, O.V. Stolbov, Yu.L. Raikher, Physical Review E92(2015) 023202.

[28] R. Rosensweig, Ferrohydrodynamics, Cambridge University Press, Cambridge, 1985.

[29] R.Balesku, Equilibrium andnon-equilibrium statistical mechanics, Wiley-Interscience publication. John Wiley \& Sons, 1975.

[30] Y.Shkel, D.Klingenberg, J.Appl.Phys. 83(1998) 7834. 
[31] M.T. Lopez-Lopez, D.Y. Borin, A.Y. Zubarev, Physical Review E96(2) (2017) 022605. 